\title{
The Liouville-type theorem for problems with nonstandard growth derived by Caccioppoli-type estimate
}

\section{Sylwia Dudek ${ }^{1}$}

Received: 6 September 2017 / Accepted: 25 February 2020 / Published online: 2 March 2020

(c) The Author(s) 2020

\begin{abstract}
Let $u$ be a nonnegative solution to the PDI $-\operatorname{div} \mathcal{A}(x, u, \nabla u) \geqslant \mathcal{B}(x, u, \nabla u)$ in $\Omega$, where $\mathcal{A}$ and $\mathcal{B}$ are differential operators with $p(x)$-type growth. As a consequence of the Caccioppoli-type inequality for the solution $u$, we obtain the Liouville-type theorem under some integral condition. We simplify the assumptions on functions $\mathcal{A}$ and $\mathcal{B}$, and we do not restrict the range of $p(x)$ by the dimension $n$, therefore we can cover quite general family of problems.
\end{abstract}

Keywords Caccioppoli inequality · Liouville-type theorem - Nonstandard growth Variable exponent Lebesgue space

Mathematics Subject Classification 26D10 · 35J60 · 35J91

\section{Introduction}

The purpose of this paper is to study some consequences of the Caccioppoli-type estimates for solutions to

$$
-\operatorname{div} \mathcal{A}(x, u, \nabla u) \geqslant \mathcal{B}(x, u, \nabla u) \text { in } \Omega,
$$

where $\mathcal{A}$ and $\mathcal{B}$ satisfy some structural conditions with respect to a variable growth exponent $p(x)$ (see (H1)-(H3) below). We assume that the function $p \in \mathcal{P}^{\log }(\Omega)$, i.e., is such that

Communicated by Adrian Constantin.

Sylwia Dudek

sylwia.dudek@pk.edu.pl

1 Department of Applied Mathematics, Faculty of Computer Science and Telecommunications, Cracow University of Technology, Warszawska 24, 31-155 Cracow, Poland 


$$
1<p^{-}:=\underset{x \in \Omega}{\operatorname{essinf}} p(x) \leqslant p(x) \leqslant p^{+}:=\underset{x \in \Omega}{\operatorname{ess} \sup } p(x)<\infty
$$

and $p$ is log-Hölder continuous or Lipschitz continuous. As a result of the Caccioppoli inequality, we obtain the Liouville-type theorem for problem (1).

We deal with the variable exponent Lebesgue and Sobolev spaces, which recently have received more and more attention both-from the theoretical and from the applied point of view. We refer to [15,31] for detailed information on the theoretical approach and to the survey [26] summarising inter alia developments on qualitative properties of solutions to the related PDEs (see also $[6,16]$ ). The typical examples of equations stated in variable exponent spaces are models of electrorheological fluids, see e.g. [36,37]. These kind of materials have been intensively investigated recently. Electrorheological fluids change their mechanical properties dramatically when an external electric field is applied, so the variable exponent Lebesgue spaces provide a natural setting for their modelling. Moreover, we find an applications of variable exponent equations in models of non-Newtonian fluid dynamics [24], image restoration processing [8], or thermistor model [41].

In this paper, we study the Caccioppoli-type inequalities which are crucial tools in investigation on quantitative properties of solutions to elliptic and parabolic problems. In the constant exponent case they are widely used among others to obtain existence, nonexistence, Liouville-type theorems [30,33], and Harnack theorems for $p$-harmonic functions [32]. The Caccioppoli-type inequalities pose cores of the regularity methods, see $[14,34]$.

The Caccioppoli-type inequalities are considered as well in the variable exponent setting. There are some results in the literature proved with the use of Caccioppoli-type inequality for weak subsolutions or supersolutions to PDEs. We would like to mention Liouville theorems [1,17], Harnack inequalities [2,4,25,27,39] and their further consequences-maximum and comparison principles [20,22]. The already mentioned applications are important in the regularity theory as some versions of the Harnack inequality imply that solutions are locally Hölder continuous [3].

The conditions sufficient to prove that solutions to certain problems are constant functions are called nonexistence results, i.e., nonexistence of nontrivial solutions, or the Liouville-type results. Despite multiple results of this type for various problems corresponding to (1) with constant (but not necessarilly power-type) growth $[7,10-$ $13,21,29,38]$, to our best knowlegde, the variable exponent versions are considered only in $[1,5,17,23,40]$. Let us point out that four of them appeared in recent two years.

In this paper, we simplify the assumptions on functions $\mathcal{A}$ and $\mathcal{B}$. Moreover, we do not restrict the range of $p(x)$ by the dimension $n$, see open problem 2 in [1]. By using new approach, we prove the Liouville-type theorem under some integral condition, see Theorem 4.1 below.

The most important example of operator $\mathcal{A}$ with $p(x)$-growth is $\mathcal{A}=|\zeta|^{p(x)-2} \zeta$. The Liouville-type theorems for problems involving such $\mathcal{A}$ are proved in e.g. [1,5]. In this papers we find diffrent approach to ours. Moreover, the authors require the $p(x)$-growth condition from above on $\mathcal{B}$. We do not impose restriction from above, so we consider more general family of problems, as for instance $\mathcal{B}(x, u, \zeta)=$ 
$c(x)\left(\kappa+|\zeta|^{2}\right)^{\frac{p(x)-2}{2}}|\zeta|^{2}$ for some $\kappa>0$ and with a measurable and bounded function $c: \Omega \rightarrow \mathbb{R}$, is allowed.

The construction used in this paper for obtaining Caccioppoli inequality is known in the constant exponent case, see e.g. $[9,35]$. There, the inequalities related to our estimates are obtained as the consequence of inequalities $\Delta_{p}\left(u^{\alpha}\right) \geqslant 0$ with a certain constant $\alpha>0$ and the inequality $-\Delta_{p} u \geqslant \Phi$ for some locally inegrable function $\Phi$. Our approach to Caccioppoli-type inequalities in variable exponent setting is new and according to our best knowledge has appeared so far only in [17]. There, the Caccioppoli inequality is applied in proving a Liouville-type theorem for PDI of the form $-\Delta_{p(x)} u \geqslant \Phi$, where function $\Phi$ is locally integrable and satisfies some compatibility condition with variable exponent $p$, while $u$ is a nonnegative weak solution. In the literature the proofs rely largely on the choice of appropriate test function for some weak formulation of considered PDIs. This approach leads to a number of difficulties, as for example we have no simple relations between the norm and the modular in variable setting. Moreover, our method of construction of the inequalities is a handy tool. Not only is it easy to conduct but also it gives deep results such as classical inequalities with the best constants [35].

The paper is organised as follows. In Sect. 2 we give some basic facts about variable exponent spaces and we establish the assumptions. Section 3 is devoted to derivation of Caccioppoli inequality for solutions to (1). In Sect. 4 we concentrate on the Liouvilletype theorems and in the Sect. 5 we present the links with the existing results.

\section{Preliminaries}

\subsection{Notation}

In what follows we assume that $\Omega \subseteq \mathbb{R}^{n}$ is an open subset not necessarily bounded. For a function $f$ defined on set $A$, by $f \chi_{A}$ we understand function $f$ extended by 0 outside $A$. Moreover, by $B(R) \subseteq \mathbb{R}^{n}$ we denote a ball centered at the origin with radius $R>0$.

\subsubsection{Variable exponent}

We suppose that a measurable function $p: \Omega \rightarrow(1, \infty)$ is such that

$$
1<p^{-}:=\underset{x \in \Omega}{\operatorname{essinf}} p(x) \leqslant p(x) \leqslant p^{+}:=\underset{x \in \Omega}{\operatorname{ess} \sup } p(x)<\infty .
$$

By $\mathcal{P}(\Omega)$ we denote the class of functions $p$ such that (3) is satisfied. Moreover, the variable exponent $p$ is said to be log-Hölder continuous, if there exists a constant $C_{l o g}>0$ such that

$$
|p(x)-p(y)| \leqslant \frac{C_{\log }}{\log \left(e+\frac{1}{|x-y|}\right)} \text { for all } x, y \in \Omega \text {. }
$$


In this paper, we study exponents $p \in \mathcal{P}(\Omega)$ which are log-Hölder continuous or Lipschitz continuous, i.e., $p \in \mathcal{P}^{\log }(\Omega)$. Both types of exponents can be extended to the whole $\mathbb{R}^{n}$ with their constants unchanged, see Proposition 4.1.7 in [15] and Theorem 6.2 in [28]. Hence, without loss of generality we assume below that variable exponents are defined in the whole $\mathbb{R}^{n}$.

Remark 2.1 We stress that we do not restrict the range of the variable exponent $p$ by the dimension $n$, see open problem 2 in [1]. Hence, we can cover more general family of problems, than we may find in the literature.

Remark 2.2 In [1], we may find an example showing that even in the relatively simple case of $p(x)$-harmonic functions on $\mathbb{R}$, i.e., $\Delta_{p(x)} u=0$, the Liouville theorem may fail to hold. The authors consider $p(x)=1+\frac{1}{1+|x|}$, and so $p(x)>1$ for all $x \in \mathbb{R}$, but unfortunately $p^{-}=1$. In such a case several theorems in the variable settings are not true. In our approach $p^{-}=1$ is also forbidden.

\subsubsection{General Lebesgue and Sobolev spaces}

By $E(\Omega)$ we denote the set of all equivalence classes of measurable real functions defined on $\Omega$ being equal almost everywhere. The variable exponent Lebesgue space is defined as

$$
L^{p(\cdot)}(\Omega)=\left\{u \in E(\Omega): \int_{\Omega}|u(x)|^{p(x)} d x<\infty\right\}
$$

equipped with the norm $\|u\|_{L^{p(\cdot)}(\Omega)}:=\inf \left\{\lambda>0: \int_{\Omega}\left|\frac{u(x)}{\lambda}\right|^{p(x)} d x \leqslant 1\right\}$.

We define the variable exponent Sobolev space $W^{1, p(\cdot)}(\Omega)$ by

$$
W^{1, p(\cdot)}(\Omega)=\left\{u \in L^{p(\cdot)}(\Omega): \nabla u \in L^{p(\cdot)}\left(\Omega ; \mathbb{R}^{n}\right)\right\},
$$

where $\nabla u$ denotes the distributional gradient, equipped with the norm $\|u\|_{W^{1, p(\cdot)}(\Omega)}=$ $\|u\|_{L^{p(\cdot)}(\Omega)}+\|\nabla u\|_{L^{p(\cdot)}(\Omega)}$.

The spaces $\left(L^{p(\cdot)}(\Omega),\|\cdot\|_{L^{p(\cdot)}(\Omega)}\right)$ and $\left(W^{1, p(\cdot)}(\Omega),\|\cdot\|_{W^{1, p(\cdot)}(\Omega)}\right)$ are separable and reflexive Banach spaces. For more detailed information we refer to $[15,18,19]$.

\subsubsection{Differential inequality and assumptions}

Throughout this paper we suppose that $\mathcal{A}: \Omega \times \mathbb{R} \times \mathbb{R}^{n} \rightarrow \mathbb{R}^{n}$ and $\mathcal{B}: \Omega \times \mathbb{R} \times \mathbb{R}^{n} \rightarrow \mathbb{R}$ are a Carathédory functions. This means that the functions $x \rightarrow \mathcal{A}(x, u, \zeta)$ and $x \rightarrow \mathcal{B}(x, u, \zeta)$ are measurable for all $(u, \zeta) \in \mathbb{R} \times \mathbb{R}^{n}$, whereas functions $(u, \zeta) \rightarrow$ $\mathcal{A}(x, u, \zeta)$ and $(u, \zeta) \rightarrow \mathcal{B}(x, u, \zeta)$ are continuous for almost all $x \in \Omega$. Moreover, let the following conditions hold for all $(x, u, \zeta) \in \Omega \times \mathbb{R} \times \mathbb{R}^{n}$ :

(H1) $|\mathcal{A}(x, u, \zeta)| \leqslant a(x)|\zeta|^{p(x)-1}$, where $a: \Omega \rightarrow \mathbb{R}$ is a measurable positive function bounded from above by $\bar{a}<\infty$. 
(H2) $\mathcal{A}(x, u, \zeta) \cdot \zeta \geqslant c(x)|\zeta|^{p(x)}$, where $c: \Omega \rightarrow \mathbb{R}$ is a measurable positive function bounded from below by $\bar{c}<\infty$.

(H3) $\mathcal{B}(x, u, \zeta) \geqslant-b(x)|\zeta|^{p(x)-1}$, where $b: \Omega \rightarrow \mathbb{R}$ is a measurable nonnegative function bounded from above by $\bar{b}<\infty$.

We recall that such functions $\mathcal{A}, \mathcal{B}$ are called of nonstandard growth or $p(\cdot)$-growth type.

We say that $u: \Omega \rightarrow \mathbb{R}$ is a weak solution to partial differential inequality $-\operatorname{div} \mathcal{A}(x, u, \nabla u) \geqslant \mathcal{B}(x, u, \nabla u)$ and we write for the simplicity that $u$ satisfy (4), if the following definition is satisfied.

Definition 2.1 Let $p \in \mathcal{P}(\Omega)$, and (H1)-(H3) hold. We say that $u \in W_{l o c}^{1, p(\cdot)}(\Omega)$ is a solution of

$$
-\operatorname{div} \mathcal{A}(x, u, \nabla u) \geqslant \mathcal{B}(x, u, \nabla u) \text { in } \Omega
$$

if for every nonnegative compactly supported $w \in W^{1, p(\cdot)}(\Omega)$, we have

$$
\int_{\Omega} \mathcal{A}(x, u, \nabla u) \cdot \nabla w d x \geqslant \int_{\Omega} \mathcal{B}(x, u, \nabla u) w d x .
$$

Remark 2.3 The most important example of function $\mathcal{A}$ with nonstandard growth is $\mathcal{A}(x, u, \zeta)=|\zeta|^{p(x)-2} \zeta$. The Liouville-type theorems for problems involving such $\mathcal{A}$ are proved in e.g. [1,5]. In this papers we find diffrent approach to ours. Futhermore, we do not assume $p(x)$-growth condition from above for operator $\mathcal{B}$. Therefore, our studies cover more general family of problems, as we may consider $\mathcal{B}(x, u, \zeta)=$ $d_{1}(x)|\zeta|^{r(x)-1}$ with $r \geqslant p$ a.e. and with a measurable and bounded function $d_{1}: \Omega \rightarrow$ $\mathbb{R}_{+}$. Moreover, we may take $\mathcal{B}(x, u, \zeta)=d_{2}(x)\left(\kappa+|\zeta|^{2}\right)^{\frac{p(x)-2}{2}}|\zeta|^{2}$ for some $\kappa>0$ and with a measurable and bounded function $d_{2}: \Omega \rightarrow \mathbb{R}_{+}$(such operators $\mathcal{B}$ are not allowed in $[1,5])$.

Remark 2.4 In order to avoid writing the long formulae, in the notation below we skip sometimes the dependence of $u$ and $p$ on $x$, i.e., $u=u(x)$ and $p=p(x)$.

\section{Caccioppoli inequality}

First we state the following useful lemmas.

Lemma 3.1 Let $p \in \mathcal{P}(\Omega), s_{1}, s_{2} \geqslant 0$ and $\tau: \Omega \rightarrow \mathbb{R}_{+}$be a positive, continuous, and bounded function. Then for a.e. $x \in \Omega$ we have

$$
s_{1} s_{2}^{p(x)-1} \leqslant \frac{1}{p(x) \tau(x)^{p(x)-1}} \cdot s_{1}^{p(x)}+\frac{p(x)-1}{p(x)} \tau(x) \cdot s_{2}^{p(x)} .
$$


Proof We apply the classical Young inequality $a b \leqslant \frac{a^{p(x)}}{p(x)}+\frac{p(x)-1}{p(x)} b^{\frac{p(x)}{p(x)-1}}$ with $a=$ $\frac{s_{1}}{\eta(x)^{p(x)-1}}, b=\left(s_{2} \eta(x)\right)^{p(x)-1}$, where $\eta(x)$ is an arbitrary, continuous, and bounded function with positive values, to get

$$
\begin{aligned}
s_{1} s_{2}^{p(x)-1} & =\left(\frac{s_{1}}{\eta^{p(x)-1}}\right)\left(s_{2} \eta\right)^{p(x)-1} \\
& \leqslant \frac{1}{p(x)}\left(\frac{s_{1}}{\eta^{p(x)-1}}\right)^{p(x)}+\frac{p(x)-1}{p(x)}\left(s_{2} \eta\right)^{(p(x)-1) \frac{p(x)}{p(x)-1}} \\
& =\frac{1}{p(x) \eta^{p(x)(p(x)-1)}} \cdot s_{1}^{p(x)}+\frac{p(x)-1}{p(x)} \eta^{p(x)} \cdot s_{2}^{p(x)} .
\end{aligned}
$$

Now, in order to get the assertion of the lemma, it suffices to substitute $\tau(x)=\eta(x)^{p(x)}$.

Lemma 3.2 Let $u \in W_{l o c}^{1, p(\cdot)}(\Omega)$ and $\phi$ be a nonnegative Lipschitz function with compact support in $\Omega$ such that $\int_{\operatorname{supp} \phi}|\nabla \phi|^{p(x)} \phi^{1-p(x)} d x<\infty$. We fix $0<\delta<K$, $\beta>0$ and denote

$$
u_{\delta, K}(x):=\min \{u(x)+\delta, K\}, G(x):=\left(u_{\delta, K}(x)\right)^{-\beta} \phi(x) .
$$

Then $u_{\delta, K} \in W_{l o c}^{1, p(\cdot)}\left(\mathbb{R}^{n}\right)$ and $G \in W^{1, p(\cdot)}(\Omega)$.

Proof By [15, Proposition 8.1.9], we obtain $u_{\delta, K} \in W_{l o c}^{1, p(\cdot)}\left(\mathbb{R}^{n}\right)$. Moreover, note that the truncated function satisfies $\delta \leqslant u_{\delta, K}(x) \leqslant K$ and therefore we have $\left(u_{\delta, K}(x)\right)^{-\beta} \in W_{l o c}^{1, p(\cdot)}\left(\mathbb{R}^{n}\right)$. Since $G$ is compactly supported, it holds that $G \in$ $W^{1, p(\cdot)}(\Omega)$.

\subsection{Derivation of a local inequality}

The fundamental step in our studies is deriving Caccioppoli-type estimates. The first result in this direction is of Caccioppoli type with respect to any nonnegative solution to (4) and holds for Lipschitz and compactly supported functions. Before we formulate the main theorem of this section with the general version of Caccioppoli inequality, we derive its local version.

Lemma 3.3 Assume that $p \in \mathcal{P}(\Omega),(H 1)-(H 3)$ hold, and nonnegative $u \in W_{l o c}^{1, p(\cdot)}(\Omega)$ satisfies (4). Assume further that $\varepsilon(x)$ is a positive, bounded, and continuous function. Moreover, there exists a parameter $\beta>0$ such that $\sigma(x):=\beta \bar{c}-2 \bar{a} \varepsilon(x) \frac{p(x)-1}{p(x)}>0$. Then, for every $0<\delta<K$, the following inequality holds for every nonnegative Lipschitz function $\phi$ with compact support in $\Omega$ 


$$
\begin{aligned}
& \int_{\Omega \cap\{u \leqslant K-\delta\}} \sigma(x)|\nabla u|^{p(x)}(u+\delta)^{-\beta-1} \phi d x \\
& \quad \leqslant \int_{\Omega \cap\{u \leqslant K-\delta\}} \frac{\bar{a}}{p(x) \varepsilon(x)^{p(x)-1}}(u+\delta)^{p(x)-\beta-1}|\nabla \phi|^{p(x)} \phi^{1-p(x)} d x \\
& \quad+\int_{\Omega \cap\{u \leqslant K-\delta\}} \frac{\bar{b}^{p(x)}}{p(x)(\bar{a} \varepsilon(x))^{p(x)-1}}(u+\delta)^{p(x)-\beta-1} \phi d x+C(\delta, K),
\end{aligned}
$$

where

$$
\begin{aligned}
C(\delta, K):= & K^{-\beta}\left[\int_{\Omega \cap\{u>K-\delta\}} \bar{a}|\nabla u|^{p(x)-1}|\nabla \phi| d x\right. \\
& \left.+\int_{\Omega \cap\{u>K-\delta\}} \bar{b}|\nabla u|^{p(x)-1} \cdot \phi d x\right] .
\end{aligned}
$$

Proof We take $w=G$, see (5), in the right-hand side of the inequality (4) and note that

$$
\begin{aligned}
\int_{\Omega} \mathcal{B}(x, u, \nabla u) G d x= & \int_{\Omega \cap\{u \leqslant K-\delta\}} \mathcal{B}(x, u, \nabla u)(u+\delta)^{-\beta} \phi d x \\
& +K^{-\beta} \int_{\Omega \cap\{u>K-\delta\}} \mathcal{B}(x, u, \nabla u) \phi d x
\end{aligned}
$$

Exploiting condition (H3), we obtain

$$
\begin{aligned}
\int_{\Omega} \mathcal{B}(x, u, \nabla u) G d x \geqslant & -\int_{\Omega \cap\{u \leqslant K-\delta\}} \bar{b}|\nabla u|^{p(x)-1}(u+\delta)^{-\beta} \phi d x \\
& -K^{-\beta} \int_{\Omega \cap\{u>K-\delta\}} \bar{b}|\nabla u|^{p(x)-1} \phi d x .
\end{aligned}
$$

On the other hand by (H1) and (H2), inequality (4) implies

$$
\begin{aligned}
\int_{\Omega} \mathcal{B}(x, u, \nabla u) G d x \leqslant & \int_{\Omega} \mathcal{A}(x, u, \nabla u) \cdot \nabla G d x \\
\leqslant & -\beta \int_{\Omega \cap\{u \leqslant K-\delta\}} \mathcal{A}(x, u, \nabla u) \cdot \nabla u(u+\delta)^{-\beta-1} \phi d x \\
& +\int_{\Omega \cap\{u \leqslant K-\delta\}}|\mathcal{A}(x, u, \nabla u)| \cdot|\nabla \phi|(u+\delta)^{-\beta} d x \\
& +K^{-\beta} \int_{\Omega \cap\{u>K-\delta\}}|\mathcal{A}(x, u, \nabla u)| \cdot|\nabla \phi| d x
\end{aligned}
$$




$$
\begin{aligned}
\leqslant & -\beta \int_{\Omega \cap\{u \leqslant K-\delta\}} \bar{c}|\nabla u|^{p(x)}(u+\delta)^{-\beta-1} \phi d x \\
& +\int_{\Omega \cap\{u \leqslant K-\delta\}} \bar{a}|\nabla u|^{p(x)-1}|\nabla \phi|(u+\delta)^{-\beta} d x \\
& +K^{-\beta} \int_{\Omega \cap\{u>K-\delta\}} \bar{a}|\nabla u|^{p(x)-1}|\nabla \phi| d x .
\end{aligned}
$$

Note that all the above integrals are finite (see Lemma 3.2, as for $0 \leqslant u \leqslant K-\delta$ we have $\delta \leqslant u+\delta \leqslant K$ ). We compute further that

$$
\begin{aligned}
& \int_{\Omega \cap\{u \leqslant K-\delta\}} \bar{a}|\nabla u|^{p(x)-1}|\nabla \phi|(u+\delta)^{-\beta} d x \\
& \quad=\int_{\operatorname{supp} \phi \cap\{u \leqslant K-\delta\}} \bar{a}|\nabla u|^{p(x)-1}\left(\frac{|\nabla \phi|}{\phi}(u+\delta)\right)(u+\delta)^{-\beta-1} \phi d x .
\end{aligned}
$$

We apply Lemma 3.1 with $s_{1}=\frac{|\nabla \phi|}{\phi}(u+\delta)$ and $s_{2}=|\nabla u|$, to get

$$
\begin{aligned}
& \int_{\Omega \cap\{u \leqslant K-\delta\}} \bar{a}|\nabla u|^{p(x)-1}|\nabla \phi|(u+\delta)^{-\beta} d x \\
& \leqslant \int_{\operatorname{supp} \phi \cap\{u \leqslant K-\delta\}} \varepsilon(x) \bar{a} \frac{p(x)-1}{p(x)}|\nabla u|^{p(x)}(u+\delta)^{-\beta-1} \phi d x \\
& \quad+\int_{\operatorname{supp} \phi \cap\{u \leqslant K-\delta\}} \frac{\bar{a}}{p(x) \varepsilon(x)^{p(x)-1}}\left(\frac{|\nabla \phi|}{\phi}\right)^{p(x)}(u+\delta)^{p(x)-\beta-1} \phi d x .
\end{aligned}
$$

Combining these estimates, we deduce that

$$
\begin{aligned}
& -\int_{\Omega \cap\{u \leqslant K-\delta\}} \bar{b}|\nabla u|^{p(x)-1} \cdot(u+\delta)^{-\beta} \phi d x \\
& \quad+\int_{\Omega \cap\{u \leqslant K-\delta\}}\left(\bar{c} \beta-\bar{a} \varepsilon(x) \frac{p(x)-1}{p(x)}\right)|\nabla u|^{p(x)}(u+\delta)^{-\beta-1} \phi d x \\
& \leqslant \int_{\operatorname{supp} \phi \cap\{u \leqslant K-\delta\}} \frac{\bar{a}}{p(x) \varepsilon(x)^{p(x)-1}}(u+\delta)^{p(x)-\beta-1}|\nabla \phi|^{p(x)} \phi^{1-p(x)} d x+C(\delta, K),
\end{aligned}
$$

where

$$
\begin{aligned}
C(\delta, K):= & K^{-\beta}\left[\int_{\Omega \cap\{u>K-\delta\}} \bar{a}|\nabla u|^{p(x)-1}|\nabla \phi| d x\right. \\
& \left.+\int_{\Omega \cap\{u>K-\delta\}} \bar{b}|\nabla u|^{p(x)-1} \cdot \phi d x\right] .
\end{aligned}
$$


Now we focus on the first integral in the above estimates. As before, we apply Lemma 3.1, but now with $s_{1}=\frac{\bar{b}}{\bar{a}}(u+\delta)$ and $s_{2}=|\nabla u|$ (with the same bounded function $\varepsilon(x)>0$ ). Note that by (H1) we have that $\bar{a}>0$. Hence, we get

$$
\begin{aligned}
& -\bar{a} \int_{\Omega \cap\{u \leqslant K-\delta\}} \frac{\bar{b}}{\bar{a}}|\nabla u|^{p(x)-1}(u+\delta) \cdot(u+\delta)^{-\beta-1} \phi d x \\
& \geqslant-\bar{a} \int_{\Omega \cap\{u \leqslant K-\delta\}} \varepsilon(x) \frac{p(x)-1}{p(x)}|\nabla u|^{p(x)}(u+\delta)^{-\beta-1} \phi d x \\
& -\int_{\Omega \cap\{u \leqslant K-\delta\}} \frac{\bar{b}^{p(x)}}{p(x)(\bar{a} \varepsilon(x))^{p(x)-1}}(u+\delta)^{p(x)-\beta-1} \phi d x .
\end{aligned}
$$

This implies that

$$
\begin{aligned}
& \int_{\Omega \cap\{u \leqslant K-\delta\}}\left(\bar{c} \beta-2 \bar{a} \varepsilon(x) \frac{p(x)-1}{p(x)}\right)|\nabla u|^{p(x)}(u+\delta)^{-\beta-1} \phi d x \\
& \leqslant \int_{\operatorname{supp} \phi \cap\{u \leqslant K-\delta\}} \frac{\bar{a}}{p(x) \varepsilon(x)^{p(x)-1}}(u+\delta)^{p(x)-\beta-1}|\nabla \phi|^{p(x)} \phi^{1-p(x)} d x \\
& \quad+\int_{\Omega \cap\{u \leqslant K-\delta\}} \frac{\bar{b}^{p(x)}}{p(x)(\bar{a} \varepsilon(x))^{p(x)-1}}(u+\delta)^{p(x)-\beta-1} \phi d x+C(\delta, K),
\end{aligned}
$$

where $C(\delta, K)$ is given by $(7)$.

\subsubsection{General version of Caccioppoli inequality}

The main goal of this section is the following result.

Theorem 3.1 (Caccioppoli estimate) Let $p \in \mathcal{P}(\Omega),(H 1)-(H 3)$ hold, and nonnegative $u \in W_{\text {loc }}^{1, p(\cdot)}(\Omega)$ satisfies (4). Assume further that $\varepsilon(x)$ is a positive, bounded, and continuous function. Moreover, there exists a parameter $\beta>0$ such that $\sigma(x):=$ $\bar{c} \beta-2 \bar{a} \varepsilon(x) \frac{p(x)-1}{p(x)}>0$. Then the following inequality holds

$$
\begin{aligned}
\int_{\Omega} \sigma(x)|\nabla u|^{p(x)} u^{-\beta-1} \phi d x \leqslant & \int_{\Omega} \frac{\bar{a}}{p(x) \varepsilon(x)^{p(x)-1}} u^{p(x)-\beta-1}|\nabla \phi|^{p(x)} \phi^{1-p(x)} d x \\
& +\int_{\Omega} \frac{\bar{b}^{p(x)}}{p(x)(\bar{a} \varepsilon(x))^{p(x)-1}} u^{p(x)-\beta-1} \phi d x
\end{aligned}
$$

where $\phi$ is a nonnegative Lipschitz function with compact support in $\Omega$ such that the integral $\int_{\text {supp } \phi}|\nabla \phi|^{p(x)} \phi^{1-p(x)} d x$ is finite.

Proof First we pass to the limit with $\delta \searrow 0$ in the local version of Caccioppoli inequality (6).

We show first that under our assumptions, when $\delta \searrow 0$, we have 


$$
\begin{gathered}
\int_{\Omega \cap\{u \leqslant K-\delta\}} \frac{\bar{a}}{p(x) \varepsilon(x)^{p(x)-1}}(u+\delta)^{p(x)-\beta-1}|\nabla \phi|^{p(x)} \phi^{1-p(x)} d x \\
\rightarrow \int_{\Omega \cap\{u \leqslant K\}} \frac{\bar{a}}{p(x) \varepsilon(x)^{p(x)-1}} u^{p(x)-\beta-1}|\nabla \phi|^{p(x)} \phi^{1-p(x)} d x
\end{gathered}
$$

for every $\phi$ as in assumptions of the theorem.

Note that $(u+\delta)^{p(x)-\beta-1} \chi_{\{u+\delta \leqslant K\}} \stackrel{\delta \searrow 0}{\rightarrow} u^{p(x)-\beta-1} \chi_{\{u \leqslant K\}}$ a.e. in $\Omega$. We show (9) independently on disjoint subsets of $\Omega$. Hence, we have

$$
\begin{aligned}
& \int_{\Omega \cap\{u \leqslant K-\delta\}} \frac{\bar{a}}{p(x) \varepsilon(x)^{p(x)-1}}(u+\delta)^{p(x)-\beta-1}|\nabla \phi|^{p(x)} \phi^{1-p(x)} d x \\
& \quad=\sum_{i=1}^{3} \int_{E_{i} \cap\{u \leqslant K-\delta\}} \frac{\bar{a}}{p(x) \varepsilon(x)^{p(x)-1}}(u+\delta)^{p(x)-\beta-1}|\nabla \phi|^{p(x)} \phi^{1-p(x)} d x,
\end{aligned}
$$

where $E_{1}=\{x \in \Omega: p(x)=\beta+1\}, E_{2}=\{x \in \Omega: p(x)<\beta+1\}, E_{3}=$ $\{x \in \Omega: p(x)>\beta+1\}$. Convergence on $E_{1}$ follows from the Lebesgue Monotone Convergence Theorem, as on this set the only expression involving $\delta$ is the characteristic function $\chi_{\{u+\delta \leqslant K\}}$.

Let us concentrate on the case when $\delta \searrow 0$ on $E_{2}$. We apply the Lebesgue Monotone Convergence Theorem as on this set

$$
(u+\delta)^{p(x)-\beta-1} \chi_{\{u+\delta \leqslant K\}} \nearrow u^{p(x)-\beta-1} \chi_{\{u \leqslant K\}} .
$$

Indeed, we note first that then for a.e. $x \in \Omega$ such that $u>0$, we have that $(u+$ $\delta)^{p(x)-\beta-1} \nearrow u^{p(x)-\beta-1} \neq 0$. Moreover, we observe that for a.e. $x \in \Omega$ we have $\chi_{\{0<u \leqslant K-\delta\}} \nearrow \chi_{\{0<u<K\}}$.

In the case of $E_{3}$, without loss of generality, we assume that $K>1$. Then we apply the Lebesgue Dominated Convergence Theorem as

$$
\begin{gathered}
\int_{E_{3} \cap\{\nabla u \neq 0\}} \frac{\bar{a}}{p(x) \varepsilon(x)^{p(x)-1}}(u+\delta)^{p(x)-\beta-1}|\nabla \phi|^{p(x)} \phi^{1-p(x)} d x \\
\leqslant K^{p^{+}-\beta-1} \frac{\bar{a} \widetilde{\varepsilon}}{p^{-}} \int_{E_{3} \cap\{u \leqslant K\}}|\nabla \phi|^{p(x)} \phi^{1-p(x)} d x<\infty,
\end{gathered}
$$

where $\widetilde{\varepsilon}=\sup _{x \in E_{3}}\left[\varepsilon(x)^{1-p(x)}\right]$. In the similar way, we can show that when $\delta \searrow 0$, we have

$$
\begin{gathered}
\int_{\Omega \cap\{u \leqslant K-\delta\}} \frac{\bar{b}^{p(x)}}{p(x)(\bar{a} \varepsilon(x))^{p(x)-1}}(u+\delta)^{p(x)-\beta-1} \phi d x \\
\rightarrow \int_{\Omega \cap\{u \leqslant K\}} \frac{\bar{b}^{p(x)}}{p(x)(\bar{a} \varepsilon(x))^{p(x)-1}} u^{p(x)-\beta-1} \phi d x .
\end{gathered}
$$

The details are left to the reader. 
To deal with the third expression on the right-hand side of (6), note that for $\delta \leqslant \frac{K}{2}$, we have

$$
\begin{aligned}
|C(\delta, K)| \leqslant & \left.\left|K^{-\beta} \int_{\Omega \cap\{u>K-\delta\}} \bar{a}\right| \nabla u\right|^{p(x)-1}|\nabla \phi| d x \mid \\
& +\left.\left|K^{-\beta} \int_{\Omega \cap\{u>K-\delta\}} \bar{b}\right| \nabla u\right|^{p(x)-1} \cdot \phi d x \mid \leqslant C(K),
\end{aligned}
$$

where

$$
C(K):=K^{-\beta}\left[\int_{\Omega \cap\left\{u \geqslant \frac{K}{2}\right\}} \bar{a}|\nabla u|^{p(x)-1}|\nabla \phi| d x+\int_{\Omega \cap\left\{u \geqslant \frac{K}{2}\right\}} \bar{b}|\nabla u|^{p(x)-1} \cdot \phi d x\right] .
$$

It suffices now to pass to the limit with $\delta \searrow 0$ on the left-hand side of (6). We assume that $\sigma(x)>0$ a.e. and thus we have $(u+\delta)^{-\beta-1} \nearrow u^{-\beta-1} \neq 0$.

Now, we pass to the limit with $K \rightarrow \infty$.

Since $|\nabla u|^{p(x)-1}|\nabla \phi|$ and $|\nabla u|^{p(x)-1} \phi$ are integrable we have $\lim _{K \rightarrow \infty} C(K)=0$. The proof is completed.

Remark 3.1 We call inequality (8) the Caccioppoli-type estimate for $u$, because it involves $|\nabla u|^{p(x)}$ on the left-hand side and $u^{p(x)}$ on the right-hand side of the inequality.

\subsubsection{Inequality for small functions}

As a consequence of Theorem 3.1, we obtain the following inequality holding for small functions, i.e., Lipschitz and compactly supported functions $\xi: \Omega \rightarrow[0,1]$. In the next section, we will apply this inequality in the main proof.

Theorem 3.2 Suppose that $p \in \mathcal{P}(\Omega)$, and (H1)-(H3) hold. Let nonnegative $u \in$ $W_{\text {loc }}^{1, p(\cdot)}(\Omega)$ satisfies (4) and assume further that there exists $\gamma>1$.

Then, for every Lipschitz function $\xi: \Omega \rightarrow[0,1]$ with compact support in $\Omega$, we have

$$
\int_{\Omega}|\nabla u|^{p(x)} u^{-\gamma} \xi^{p^{+}} d x \leqslant \bar{c} \int_{\Omega} u^{p(x)-\gamma}\left(|\nabla \xi|^{p(x)}+1\right) \chi_{\{\xi \in(0,1]\}} d x
$$

where $\bar{c}:=\max \left\{\widetilde{\varepsilon} \frac{\bar{a}\left(p^{+}\right)^{p^{+}}}{\sigma^{-} p^{-}}, \frac{\bar{a} \widetilde{\varepsilon}}{p^{-} \sigma^{-}} \sup _{x \in \Omega}\left[\left(\frac{\bar{b}}{\bar{a}}\right)^{p(x)}\right]\right\}$, with $\widetilde{\varepsilon}:=\sup _{x \in \Omega} \varepsilon(x)^{1-p(x)}$ and $\sigma^{-}:=\inf _{x \in \Omega}\left[\bar{c} \beta-2 \bar{a} \varepsilon(x) \frac{p(x)-1}{p(x)}\right]$.

Proof We apply Theorem 3.1 with $\phi(x)=\xi(x)^{p^{+}}$. Then, $\nabla \phi=p^{+} \xi^{p^{+}-1} \nabla \xi$. So

$$
\begin{aligned}
|\nabla \phi|^{p(x)} \phi^{1-p(x)} & =\left(p^{+}\right)^{p(x)} \xi^{p(x)\left(p^{+}-1\right)}|\nabla \xi|^{p(x)} \xi^{p^{+}(1-p(x))} \\
& =\left(p^{+}\right)^{p(x)} \xi^{p^{+}-p(x)}|\nabla \xi|^{p(x)}
\end{aligned}
$$


Since $\xi \in[0,1]$, we have $|\nabla \phi|^{p(x)} \phi^{1-p(x)} \leqslant\left(p^{+}\right)^{p^{+}}|\nabla \xi|^{p(x)} \chi_{\{\xi \in(0,1]\}}$.

Let $\beta+1=\gamma$. Then $\gamma>1$ and by Caccioppoli inequality (8), we get

$$
\begin{aligned}
\int_{\Omega}|\nabla u|^{p(x)} u^{-\gamma} \xi^{p^{+}} d x \leqslant & \frac{1}{\sigma^{-}}\left[\int_{\Omega} \frac{\bar{a}}{p(x) \varepsilon(x)^{p(x)-1}} u^{p(x)-\gamma}\left(p^{+}\right)^{p^{+}}|\nabla \xi|^{p(x)} \chi_{\{\xi \in(0,1]\}} d x\right. \\
& \left.+\int_{\Omega} \frac{\bar{b}^{p(x)}}{p(x)(\bar{a} \varepsilon(x))^{p(x)-1}} u^{p(x)-\gamma} \xi^{p^{+}} d x\right],
\end{aligned}
$$

where $\sigma^{-}:=\inf _{x \in \Omega}\left[\bar{c} \beta-2 \bar{a} \varepsilon(x) \frac{p(x)-1}{p(x)}\right]>0$. Function $\xi \in[0,1]$, so

$$
\int_{\Omega}|\nabla u|^{p(x)} u^{-\gamma} \xi^{p^{+}} d x \leqslant \bar{c} \int_{\Omega} u^{p(x)-\gamma}\left(|\nabla \xi|^{p(x)}+1\right) \chi_{\{\xi \in(0,1]\}} d x,
$$

where $\bar{c}:=\max \left\{\widetilde{\varepsilon} \frac{\bar{a}\left(p^{+}\right)^{p^{+}}}{\sigma^{-} p^{-}}, \frac{\bar{a} \widetilde{\varepsilon}}{p^{-} \sigma^{-}} \sup _{x \in \Omega}\left[\left(\frac{\bar{b}}{\bar{a}}\right)^{p(x)}\right]\right\}$ with $\widetilde{\varepsilon}:=\sup _{x \in \Omega} \varepsilon(x)^{1-p(x)}$.

\section{Liouville-type theorem}

In this section, we prove the Liouville-type theorem, i.e., sufficient conditions under which any nonnegative weak solution $u$ to $-\operatorname{div} \mathcal{A}(x, u, \nabla u) \geqslant \mathcal{B}(x, u, \nabla u)$ has to be a constant function. In further part of this section we show several applications of the main theorem.

Recall that $B(R) \subseteq \mathbb{R}^{n}$ denotes the ball centered at the origin with radius $R>0$.

Theorem 4.1 (Liouville-type theorem) Let as assume that $p \in \mathcal{P}^{\log }\left(\mathbb{R}^{n}\right),(H 1)$-(H3) hold, and nonnegative $u \in W_{l o c}^{1, p(\cdot)}\left(\mathbb{R}^{n}\right)$ satisfies (4). Assume further that there exists $\gamma>1$ such that

$$
\int_{B(R+1) \backslash B(R)} u^{p(x)-\gamma} d x \stackrel{R \rightarrow \infty}{\longrightarrow} 0 .
$$

Then $u$ is a constant function.

Proof We consider a sequence of Lipschitz compactly supported functions $\left\{\xi_{R}\right\}_{R \in \mathbb{N}_{+}}$ defined as follows

$$
\xi_{R}= \begin{cases}1 & \text { for } x \in B(R), \\ R+1-|x| & \text { for } x \in B(R+1) \backslash B(R), \\ 0 & \text { for } x \notin B(R+1) .\end{cases}
$$

We apply Theorem 3.2 with constant $\bar{c}>0$, and obtain

$$
\int_{\mathbb{R}^{n}}|\nabla u|^{p(x)} u^{-\gamma}\left|\xi_{R}\right|^{p^{+}} d x \leqslant \bar{c} \int_{\mathbb{R}^{n}} u^{p(x)-\gamma}\left(\left|\nabla \xi_{R}\right|^{p(x)}+1\right) \chi_{\left\{\xi_{R} \in(0,1]\right\}} d x .
$$


Observe that

$$
\int_{\mathbb{R}^{n}} u^{p(x)-\gamma}\left(\left|\nabla \xi_{R}\right|^{p(x)}+1\right) \chi_{\left\{\xi_{R} \in(0,1]\right\}} d x \leqslant 3 \int_{B(R+1) \backslash B(R)} u^{p(x)-\gamma} d x .
$$

Now, let us concentrate on the left-hand side of the considered inequality. We note that for every $R \in \mathbb{N}_{+}$we have

$$
0 \leqslant \int_{B(R)}|\nabla u|^{p(x)} u^{-\gamma} d x \leqslant \int_{\mathbb{R}^{n}}|\nabla u|^{p(x)} u^{-\gamma}\left|\xi_{R}\right|^{p^{+}} d x .
$$

To sum up, we obtain

$$
0 \leqslant \int_{B(R)}|\nabla u|^{p(x)} u^{-\gamma} d x \leqslant 3 \bar{c} \int_{B(R+1) \backslash B(R)} u^{p(x)-\gamma} d x .
$$

Thus, by letting $R \rightarrow \infty$, we get by assumption (11) that $|\nabla u|^{p(x)} u^{-\gamma} \equiv 0$ a.e.

Therefore $|\nabla u|^{p(x)} \equiv 0$ a.e. and we conclude that $u$ has to be a constant function, which finishes the proof.

Remark 4.1 The sufficient condition for (11) is that for $\gamma>1$, we have

$$
\int_{\mathbb{R}^{n}} u^{p(x)-\gamma} d x<\infty
$$

To highlight the significance of the main result, we present some of its direct conclusions for the $p(x)$-Laplace equation $-\Delta_{p(x)} u \geqslant 0$.

Corollary 4.1 Let $p \in \mathcal{P}^{\log }\left(\mathbb{R}^{n}\right)$ and $u \in W_{\text {loc }}^{1, p(\cdot)}\left(\mathbb{R}^{n}\right)$ be a nonnegative weak solution to $-\Delta_{p(x)} u \geqslant 0$. Assume further that there exists $\gamma>1$ such that

$$
\int_{B(R+1) \backslash B(R)} u^{p(x)-\gamma} d x \stackrel{R \rightarrow \infty}{\longrightarrow} 0 .
$$

Then $u$ is a constant function.

Moreover, let us consider nonnegative solutions to the nonlinear eigenvalue problem $-\Delta_{p(x)} u \geqslant \lambda|u|^{q(x)-2} u$, where $1<q(x)<\infty$. We get the following corollary.

Corollary 4.2 Let $p \in \mathcal{P}^{\log }\left(\mathbb{R}^{n}\right)$ and $u \in W_{\text {loc }}^{1, p(\cdot)}\left(\mathbb{R}^{n}\right)$ be a nonnegative weak solution to $-\Delta_{p(x)} u \geqslant \lambda|u|^{q(x)-2} u$ with $1<q(x)<\infty$. Assume further that there exists $\gamma>1$ such that

$$
\int_{B(R+1) \backslash B(R)} u^{p(x)-\gamma} d x \stackrel{R \rightarrow \infty}{\longrightarrow} 0 .
$$

Then $u$ is a constant function. 
Let us emphasize that Theorem 4.1 is new even in the constant exponent case, see the following corollary for the $p$-harmonic equation.

Corollary 4.3 Let $p \in(1, \infty)$ and $u \in W_{\text {loc }}^{1, p}\left(\mathbb{R}^{n}\right)$ is a nonnegative weak solution to $-\Delta_{p} u \geqslant 0$. Assume further that there exists $\gamma>1$ such that

$$
\int_{B(R+1) \backslash B(R)} u^{p-\gamma} d x \stackrel{R \rightarrow \infty}{\longrightarrow} 0 .
$$

Then $u$ is a constant function.

Remark 4.2 In the classical setting, if $\mathcal{B} \equiv 0, \mathcal{A}=\nabla u$ and $p \equiv 2$, i.e.,

$$
-\Delta u \equiv 0 \quad \text { in } \mathbb{R}^{n}
$$

then it is known that every nonconstant solution satisfies $u(x) \geqslant c|x|^{2-n}$. The requirement of faster decreasing rate implies $u \equiv 0$. Our result gives worse estimate on the growth. The best exponent that we can obtain is $1-n$, so this method is not sharp in general, see Remarks 12 and 13 in [17].

\section{Links with the existing results}

The conditions for the Liouville-type results for equations yielded in variable exponent settings have not yet been studied systematically in the literature. To our best knowledge, there exist five papers which are in variable settings, see $[1,5,17,23,40]$, and only in $[1,5]$ we find the inequality in general form with operators satisfying some structural conditions with respect to $p(x)$-growth.

The results of Wang [40] differ for ours and the rest of the mentioned papers, because the author considers problems defined on Riemannian manifolds. Nevertheless, a growth condition is crucial to prove the Liouville-type theorem in that setting as well.

In [23] the authors study problems in the form

$$
-\Delta_{p(x)} u \geqslant u^{q(x)} g(x), \quad-\Delta_{p(x)} u \geqslant|\nabla u|^{q(x)} g(x)
$$

with the specified function $g$, as well as systems of such problems and the corresponding parabolic ones, in $\mathbb{R}^{n}$ and in bounded domains. They formulate an integral condition for certain expressions involving exponents, implying nonexistence results.

In [17], we consider problems of the form

$$
-\Delta_{p(x)} u \geqslant \Phi(x, u(x), \nabla u(x)) \text { in } \Omega
$$

with nonnegative $u \in W_{l o c}^{1, p(\cdot)}(\Omega)$ and $\Phi \in L_{l o c}^{1}(\Omega)$. We consider the class $\mathcal{P}(\Omega)$ of bounded, measurable, variable exponents $p$, such that $p \in W_{l o c}^{1,1}(\Omega)$ and $|\nabla p|^{p} \in$ $L_{l o c}^{1}(\Omega)$. As for the growth of $\Phi$ we assume that 


$$
\Phi \cdot u+\sigma(x)|\nabla u|^{p(x)} \geqslant 0 \text { a.e. in } \Omega
$$

where $\sigma(\cdot)$ is bounded and continuous. These assumptions together with

$$
\lim _{R \rightarrow \infty} \int_{B(R+1) \backslash B(R)} u^{p(x)-\beta-1}\left(p(x)^{p(x)}+|\nabla p(x)|^{p(x)}\right) d x=0,
$$

imply the Liouville-type theorem for (15).

The Liouville-type theorem for quasilinear elliptic equations in $\mathbb{R}^{n}$ with variable exponent appears also in [5], where the following equation is investigated

$$
-\operatorname{div} \mathcal{A}(x, \nabla u)+\mathcal{B}(x, u)=0
$$

with operators $\mathcal{A}, \mathcal{B}$ satisfying some structure variable assumptions and $p \in \mathcal{P}\left(\mathbb{R}^{n}\right)$ such that for every $x \in \mathbb{R}^{n}$, the function $p$ is differentiable and $|\nabla p(\cdot)|$ is globally bounded. $\mathcal{A}$ and $\mathcal{B}$ one assumes

$$
\liminf _{R \rightarrow \infty}\left(R^{-2 p(x)} M(R)\right)=0 \text { for } x \in \mathbb{R}^{n},
$$

where $M(R)$ depends on $p, \nabla p, \mathcal{A}$ and some other compatibility functions and parameters.

The most general of these results are proven in [1], where the authors provide some Liouville-type theorems for $\mathcal{A}$-harmonic problems, for $(\mathcal{A}, \mathcal{B})$-harmonic equations and inequalities. In [1], a function $u \in W_{\text {loc }}^{1, p(\cdot)}(\Omega)$ is called $(\mathcal{A}, \mathcal{B})$-solution $((\mathcal{A}, \mathcal{B})$ supersolution) of

$$
-\operatorname{div} \mathcal{A}(x, u, \nabla u)=\mathcal{B}(x, u, \nabla u),
$$

if

$$
\int_{\Omega} \mathcal{A}(x, u, \nabla u) \cdot \nabla w d x=(\geqslant) \int_{\Omega} \mathcal{B}(x, u, \nabla u) w d x
$$

for all (nonnegative) $w \in \mathcal{C}_{0}^{\infty}(\Omega)$, respectively. The admissible variable exponents are such that $p \in \mathcal{P}^{\log }(\Omega)$. Moreover, the authors put additional restriction on the variable exponent as $p^{-}>n$ (see Theorem 5 in [1]) or $p^{-}<n$ (see Theorem 6 in [1]). See also condition (17) in Theorem 5.1 below.

The authors impose more restricted assumptions on $\mathcal{A}$ and $\mathcal{B}$ than we need here. They assume conditions similar to (H1)-(H3), and moreover, they assume the growth condition from the above on operator $\mathcal{B}$.

Let us mention the following result of [1].

Theorem 5.1 ([1, Theorem 9]) Suppose that there exist $\delta>0, \gamma<0$ and a divergence increasing sequence $\left\{R_{k}\right\}_{k=1}^{\infty}$ such that

$$
p_{B\left(2 R_{k}\right) \backslash B\left(R_{k}\right)}^{-}:=\operatorname{essinf}_{x \in B\left(2 R_{k}\right) \backslash B\left(R_{k}\right)} p(x) \geqslant n+\delta+1-\gamma .
$$


Additionally, let us assume that $\mathcal{B}(\cdot, u(\cdot), \nabla u(\cdot)) u^{\gamma} \in L^{1}\left(\mathbb{R}^{n}\right)$ as a function of $x \in \mathbb{R}^{n}$ and satisfies the following integral condition

$$
\int_{B(R)} \mathcal{B}(x, u(x), \nabla u(x)) u(x)^{\gamma} d x \stackrel{R \rightarrow \infty}{\longrightarrow} 0 .
$$

$$
\text { If } u>0 \text { is a bounded }(\mathcal{A}, \mathcal{B}) \text {-solution of }(16) \text { in } \mathbb{R}^{n} \text {, then } u=\text { const. }
$$

For $(\mathcal{A}, \mathcal{B})$-harmonic inequalities, the authors investigate the special kind of problems, i.e. with $\mathcal{B}=f(u)$, and then the Riccati-type inequalities with $\mathcal{B}=f(|\nabla u|)$, where $f: \mathbb{R} \rightarrow \mathbb{R}_{+}$is a nonnegative, continuous function, see Theorems 10 and 13 in [1].

We point out that (18), as well as (14), requires integrability at infinity of $u$.

Open Access This article is licensed under a Creative Commons Attribution 4.0 International License, which permits use, sharing, adaptation, distribution and reproduction in any medium or format, as long as you give appropriate credit to the original author(s) and the source, provide a link to the Creative Commons licence, and indicate if changes were made. The images or other third party material in this article are included in the article's Creative Commons licence, unless indicated otherwise in a credit line to the material. If material is not included in the article's Creative Commons licence and your intended use is not permitted by statutory regulation or exceeds the permitted use, you will need to obtain permission directly from the copyright holder. To view a copy of this licence, visit http://creativecommons.org/licenses/by/4.0/.

\section{References}

1. Adamowicz, T., Gorka, P.: The Liouville theorems for elliptic equations with nonstandard growth. Commun. Pure Appl. Anal. 14(6), 2377-2392 (2015)

2. Adamowicz, T., Hästö, P.: Harnack's inequality and the strong $p(\cdot)$-Laplacian. J. Differ. Equ. 250(3), 1631-1649 (2011)

3. Alkhutov, Y.: The Harnack inequality and the Hölder property of solutions of nonlinear elliptic equations with nonstandard growth condition, Differ. Uravn. 33(12), 1651-1660 (1997), 1726. [Translation in Differential Equations 33 (12) (1997), 1653-1663.]

4. Baalal, A., Qabil, A.: Harnack inequality nad continuity of solutions for quasilinear elliptic equations in Sobolev spaces with variable exponent. Nonlinear Anal. Differ. Equ. 2, 69-81 (2014)

5. Baalal, A.: Liouville-type result for quasilinear elliptic problems with variable exponent. Int. J. Pure Appl. Math. 104(1), 57-68 (2015)

6. Barnaś, S.: Existence results for hemivariational inequality involving $p(x)$-Laplacian. Opuscula Math. 32, 439-454 (2012)

7. Caristi, G., Mitidieri, E.: Some Liouville theorems for quasilinear elliptic inequalities. Doklady Math. 79(1), 118-124 (2009)

8. Chen, Y., Levine, S., Rao, M.: Variable exponent, linear growth functionals in image restoration. SIAM J. Appl. Math. 66(4), 1383-1406 (2006)

9. D’Ambrosio, L.: Hardy type inequalities related to degenerate elliptic differential operators. Ann. Sc. Norm. Super. Pisa Cl. Sci. 5(4), 451-486 (2005)

10. D'Ambrosio, L.: Liouville theorems for anisotropic quasilinear inequalities. Nonlinear Anal. TMA 70(8), 2855-2869 (2009)

11. D'Ambrosio, L., Mitidieri, E.: A priori estimates and reduction principles for quasilinear elliptic problems and applications. Adv. Differ. Equ. 17(9-10), 935-1000 (2012)

12. D'Ambrosio, L., Mitidieri, E.: A priori estimates, positivity results, and nonexistence theorems for quasilinear degenerate elliptic inequalities. Adv. Math. 224(3), 967-1020 (2010)

13. D'Ambrosio, L., Mitidieri, E.: Liouville theorems for elliptic systems and applications. J. Math. Anal. Appl. 413(1), 121-138 (2014) 
14. DiBenedetto, E.: Degenerate Parabolic Equations. Universitext. Springer, New York (1993)

15. Diening, L., Harjulehto, P., Hästö, P., Ružička, M.: Lebesgue and Sobolev Spaces with Variable Exponents, vol. 2017. Springer, Heidelberg (2011). Lecture Notes in Math

16. Dudek, S.: Nontrivial solution for nonlinear $p(x)$-Laplacian Dirichlet problem with the sign-changing weight. Dyn. Syst. Appl. 24, 67-81 (2015)

17. Dudek, S., Skrzypczak, I.: Liouville theorems for elliptic problems in variable exponent spaces. Commun. Pure Appl. Anal. 16(2), 513-532 (2017)

18. Fan, X., Zhao, D.: On the generalized Orlicz-Sobolev space $W^{k, p(x)}(\Omega)$. J. Gansu Educ. Coll. 12(1), 1-6 (1998)

19. Fan, X., Zhao, D.: On the spaces $L^{p(x)}(\Omega)$ and $W^{m, p(x)}(\Omega)$. J. Math. Anal. Appl. 263, 424-446 (2001)

20. Fan, X., Zhao, Y., Zhang, Q.: A strong maximum principle for $p(x)$-Laplace equations. Chin. J. Contemp. Math. 24(3), 277-282 (2003). [Translation of Chinese Ann. Math. Ser. A 24 (4) (2003), 495-500.]

21. Filippucci, R.: Nonexistence of positive weak solutions of elliptic inequalities. Nonlinear Anal. TMA 70(8), 2903-2916 (2009)

22. Fortini, R., Mugnai, D., Pucci, P.: Maximum principles for anisotropic elliptic inequalities. Nonlinear Anal. 70(8), 2917-2929 (2009)

23. Galakhov, E., Salieva, O., Uvarova, L.: Nonexistence results for some nonlinear inequalities with functional parameters. Electron. J. Qual. Theory Differ. Equ. 85, 1-11 (2015)

24. Gwiazda, P., Świerczewska-Gwiazda, A., Wróblewska-Kamińska, A.: Monotonicity methods in generalized Orlicz spaces for a class of non-Newtonian fluids. Math. Methods Appl. Sci. 33(2), 125-137 (2010)

25. Harjulehto, P., Hästö, P., Latvala, V.: Harnack's inequality for $p(\cdot)$-harmonic functions with unbounded exponent $p$. J. Math. Anal. Appl. 352, 345-359 (2009)

26. Harjulehto, P., Hästö, P., Lê, U., Nuortio, M.: Overview of differential equations with non-standard growth. Nonlinear Anal. 72, 4551-4574 (2010)

27. Harjulehto, P., Kinnunen, J., Lukkari, T.: Unbounded supersolutions of nonlinear equations with nonstandard growth. Bound. Value Probl. (2007). https://doi.org/10.1155/2007/48348

28. Heinonen, J.: Lectures on Analysis on Metric Spaces. Universitext. Springer, New York (2001)

29. Kałamajska, A., Pietruska-Pałuba, K., Skrzypczak, I.: Nonexistence results for differential inequalities involving A-Laplacian. Adv. Differ. Equ. 17(3-4), 307-336 (2012)

30. Kawohl, B., Kurta, V.: A Liouville comparison principle for solutions of singular quasilinear elliptic second-order partial differential inequalities. Commun. Pure Appl. Anal. 10, 1747-1762 (2011)

31. Kovácik, O., Rákosník, J.: On spaces $L^{p(x)}$ and $W^{1, p(x)}$. Czechoslovak Math. J. 41(116), 592-618 (1991)

32. Lindquist, P.: Notes on the $p$-Laplace equation. www.math.ntnu.no/ lqvist/p-laplace.pdf

33. Mitidieri, E., Pokhozhaev, S.I.: Some generalizations of the Bernstein's theorem. Differ. Equ. 38(3), 392-397 (2002)

34. Moser, J.: On Harnack's theorem for elliptic differential equations. Commun. Pure Appl. Math. 14, 577-591 (1961)

35. Skrzypczak, I.: Hardy-type inequalities derived from $p$-harmonic problems. Nonlinear Anal. TMA 93, 30-50 (2013)

36. Rajagopal, K., Ružička, M.: On the modeling of electrorheological materials. Mech. Res. Commun. 23, 401-407 (1996)

37. Ružička, M.: Electrorheological Fluids: Modeling and Mathematical Theory. Springer, Berlin (2000)

38. Serrin, J.: The Liouville theorem for homogeneous elliptic differential inequalities. Problems in mathematical analysis. J. Math. Sci. (N. Y.) 179(1), 174-183 (2011)

39. Toivanen, O.: Harnack's inequality for general solutions with nonstandard growth. Ann. Acad. Sci. Fenn. Math. 37(2), 571-577 (2012)

40. Wang, L.F.: Liouville theorem for the variable exponent Laplacian. Chin. J. East China Norm. Univ. Natur. Sci. Ed. 1, 84-93 (2009)

41. Zhikov, V.V.: On some variational problems. Russ. J. Math. Phys. 5(1), 105-116 (1998)

Publisher's Note Springer Nature remains neutral with regard to jurisdictional claims in published maps and institutional affiliations. 